\title{
The design and obstacle-overcoming analysis of multiphase connecting- rod wheeled robot
}

\author{
Chen-yang Shen ${ }^{1, a}$, Yu-jun Wang ${ }^{2, b}$, Can Fang ${ }^{3, c}$, Peng-fei Yin ${ }^{4, d}$ and Jie \\ Huang ${ }^{5 . e}$ \\ ${ }^{1,2,3,4,5}$ School of Computer and Information Science, Southwest University, Chongqing, China \\ a985316966@qq.com, b774872400@qq.com, c1007636983@qq.com
}

\begin{abstract}
Keywords: multiphase connecting-rod structure; singularity phenomenon; obstacle-overcoming;
\end{abstract}
\begin{abstract}
This article is mainly aimed at the solving the problem of singularity phenomenon which exists in the connecting-rod wheeled robot. We propose a multiphase connecting-rod wheeled robot based on multiphase connecting-rod structure and analyze the design principle and obstacle-overcoming ability of the robot. By conducting experiments, we proved that the robot can resolve the singularity phenomenon which appears in the connecting-rod wheeled robot and its obstacle-overcoming performance and movement stability is well. We expect the robot can be applied to the exploration and rescue environments.
\end{abstract}

\section{Introduction}

With the human exploration in uncharted territory, the human working environment has become more unstructured. In order to adapt to these changes, it demands the robot to accommodate different environments. For this reason, the small mobile robots become an important choice. This kind of robot has the characters of high reliability, strong adaptability, strong concealment, which makes it become the ideal platform of carrying out dangerous tasks [1]. These robots have been widely applied to various military fields, and are faced with a lot of unknown terrain. Therefore, improving the terrain adaptability and obstacle-overcoming ability of mobile robot has already become an important direction in research.

In order to improve the terrain adaptability and the obstacle-overcoming ability of the robot, Junke Li who is in the southwest university has put forward a kind of wheel robot. It was named connecting-rod wheeled robot [2]. Using connecting-rod as its walking mechanism, and this mechanism takes advantage of the principle of power transmission of four-bar-mechanism [3,4]. The robot can improve the walking efficiency and obstacle-overcoming ability, but it appears singularity phenomenon in the process of movement. The existence of this phenomenon makes the efficiency and obstacle-overcoming ability greatly reduced, and in order to effectively solve this problem, this paper proposes a multiphase connecting-rod structure as the robot's walking mechanism. We analyze the design principle and obstacle-overcoming ability of the robot, and by conducting experiments, it has proved that the multiphase connecting-rod wheeled robot can effectively solve the singularity phenomenon problem in the connecting-rod driving structure, and can be self-adapted terrain very well.

\section{Analysis of singularity phenomenon}

Under the normal circumstances of connecting-rod wheeled robot, the connecting-rod always parallels to the level of wheel center attachment, as shown in Fig. 1(a), and when the straight rod move to the same level of wheel axis hole, as shown in Fig. 1(b), The wheels of robot may occur the phenomenon of clamping as the connecting-rod position abnormalities. As shown in Fig. 1(c). The angle between the rod and the ground is acute, and because the connecting-rod is a rigid material, the motor will be blocked. This phenomenon is defined as singularity phenomenon. 


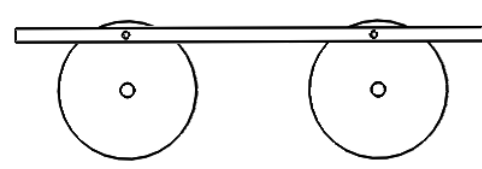

(a)

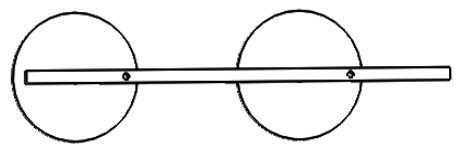

(b)

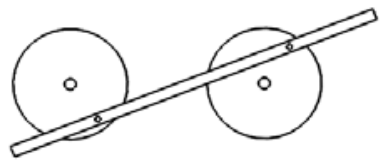

(c)

Fig. 1 connecting-rod structure

The reason of the singularity phenomenon can be analyzed as follows. We can view the movement of the connecting-rod wheeled robot as the reciprocating movement of the four-bar-mechanism. As shown in Fig. 2(a), AB is the driving part of crank; $B C$ is the connecting-rod; $\mathrm{CD}$ is the follower; $\mathrm{AD}$ is stationary. Due to the motor driver makes AB's movement actively, $\mathrm{AB}$ moves the follower $\mathrm{CD}$ through the connecting-rod $\mathrm{BC}$. The equation [5] for the freedom of four-bar-mechanism is as follows.

$$
F=3 n-2 P_{L}-P_{H}
$$

In the Eq. $1, n$ is the number of active part; $\mathrm{P}_{\mathrm{L}}$ is the number of lower pair; $\mathrm{P}_{\mathrm{H}}$ is the number of higher pair; the mechanism's degree of freedom is 1 . It is the number of driving part and the mechanism has certain movement.

When the four-bar-mechanism moves to the location of Fig. 2(b), and AB, BC, CD is collinear right now. In this location, the force of the driving part which acts on $\mathrm{CD}$ just gets through the follower CD's center, and the driving torque is 0 , so in theory it cannot push CD's rotation. The mechanism in this location is called the dead point[5]. Agency in the dead point position can be influenced by the external force, and the movement of connecting-rod mechanism is uncertain. As shown in Fig. 2(c).
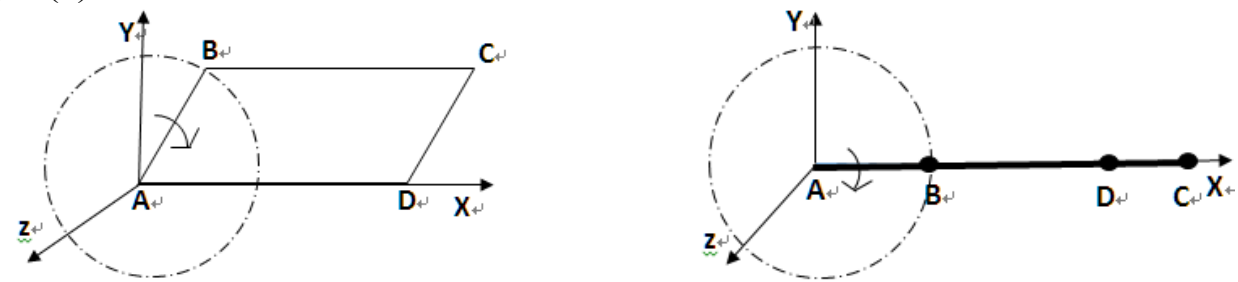

(a) (b)

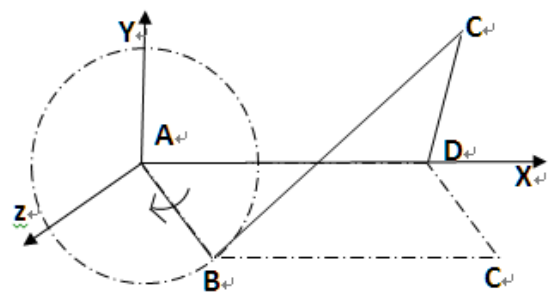

(c)

Fig. 2 four-bar-mechanism

\section{Design of multiphase connecting-rod wheeled robot}

The proposed multiphase connecting-rod wheeled robot adopts a symmetric structure and is consisted of two sets of multiphase connecting-rod which are in the same size. As shown in the Fig. 3. Its body is designed as the rectangular frame, and installed four same type motors. The battery and circuit are put in its body; 3D model of the robot is shown in Fig. 4. In the model, we assume the big circle's radius is R; the small circle's radius is $r$; the front end's length of the connecting-rod 
is L; the barrier's height is H; In order to make the robot freely move and not be hindered by obstacles, the parameter of the robot should meet the equation as follows.

$$
\left\{\begin{array}{l}
r<R \\
\left(R_{\max }=R+e\right)>H \\
R \leq L \leq 2 R
\end{array}\right.
$$

We conduct experiments and use the standard stairs to test its obstacle-overcoming ability. The stairs' parameter is as follows. The height of stair is $15.5 \mathrm{~cm}$, and long is $28.5 \mathrm{~cm}$. The parameter of physical prototype is follows. The wheel's radius is $15 \mathrm{~cm}$, and small wheel's radius is $8 \mathrm{~cm}$. the eccentric distance is $12 \mathrm{~cm}$. the connecting-rod's length is $67 \mathrm{~cm}$. Connecting-rod's front-end is $20 \mathrm{~cm}$. physical prototype is shown in Fig. 5.

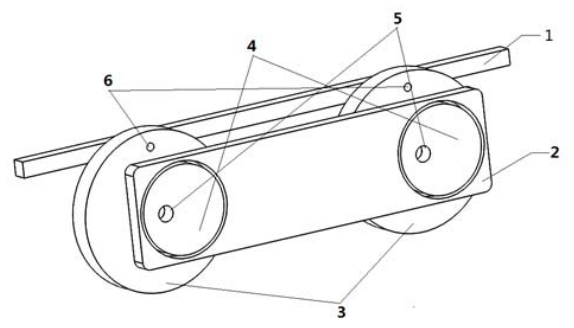

Fig. 3 multiphase connecting-rod structure

1: front end;2: sheet;3: big circle round;4: small circle round;5: axis;6: passive joint;

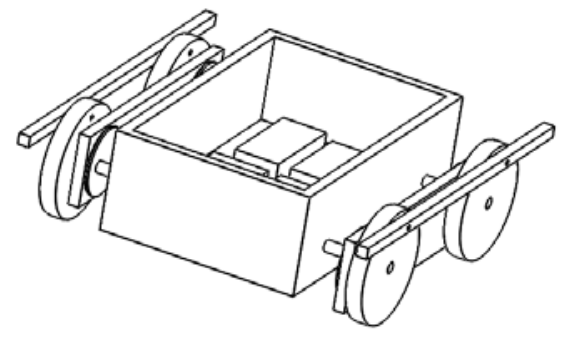

Fig. 4 3D model

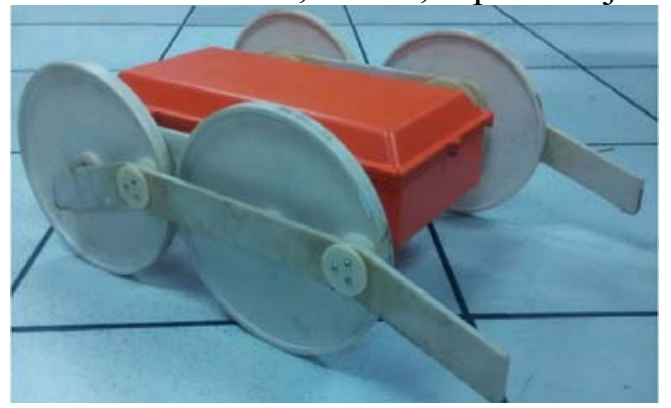

Fig. 5 physical model

\section{Experiments}

\subsection{Obstacle overcoming}

When a barrier is found in the smooth terrain, the connecting-rod puts on the obstacles, and the motor continues to drive wheels do eccentric exercise around the passive joint. Because the front and back wheel do the same movement, the front wheel's rotation will continue to drive the back wheel's rotation. When the two wheels overcome the obstacle, the robot completes the whole process of the obstacle-overcoming. The process is shown in Fig. 6.

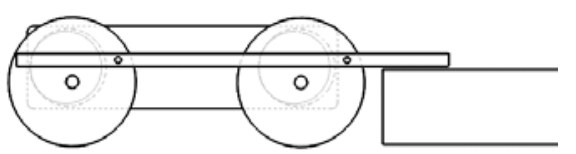

(a) (b)

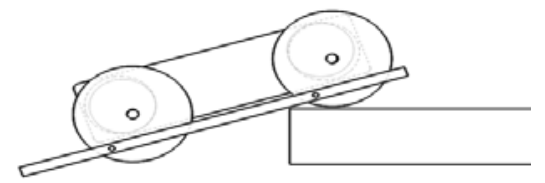

(b) (d)
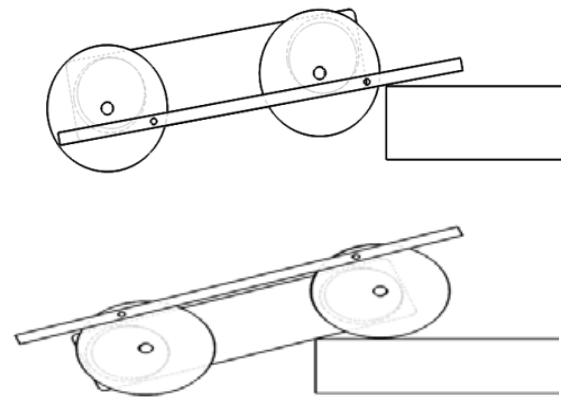


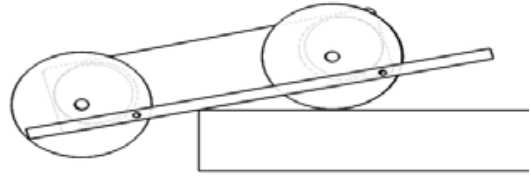

(e)

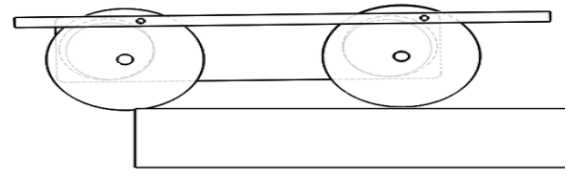

(f)

\subsection{Stairs climbing}

Fig. 6 obstacle-overcoming locomotion

The robot's stair-climbing experiment is shown in Fig. 7. The robot's stair-climbing process can be divided into three steps.

In the first, when the height of the stairs satisfies the Eq. 2, the robot's front end of the connecting-rod has an opportunity to put on the stairs, as shown in Fig. 7(a)(b). Second, after the robot's front end putting on the stair, the front wheel and back wheel will make the same eccentric exercise around the passive joint. As shown in Fig. 7(c)(d). Finally, when the wheels contact to the surface of next stair, the connecting-rod will leave the stair and do the movement with the wheels. Until the front end of the connecting-rod put on the next stair, thus the first cycle of movement is completed. As shown in Fig. 7(e)(f). After that the robot will do the repeat movement until it finishes all stairs.

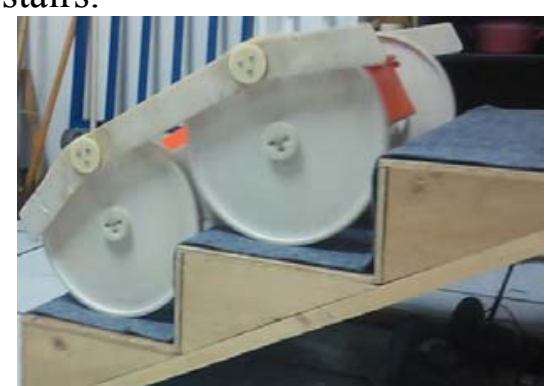

(a)

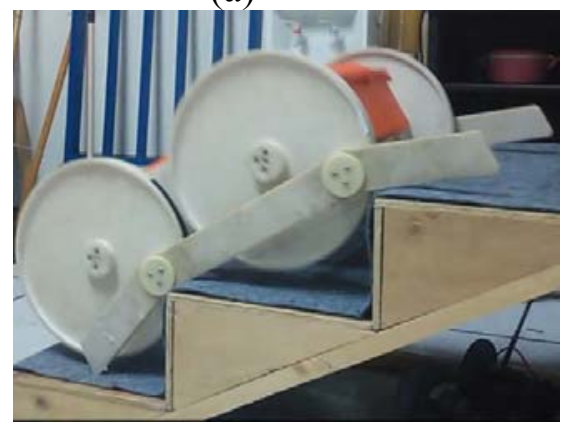

(c)

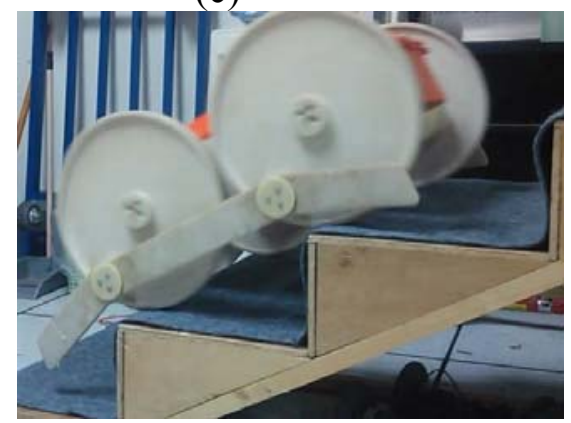

(e)

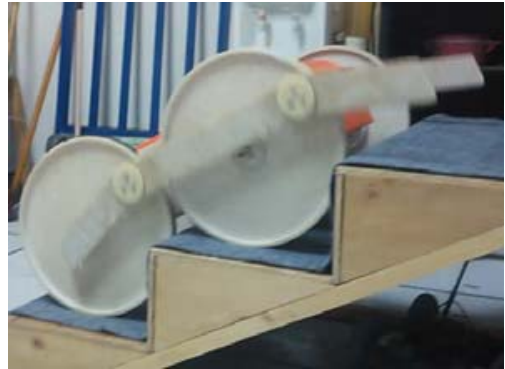

(b)

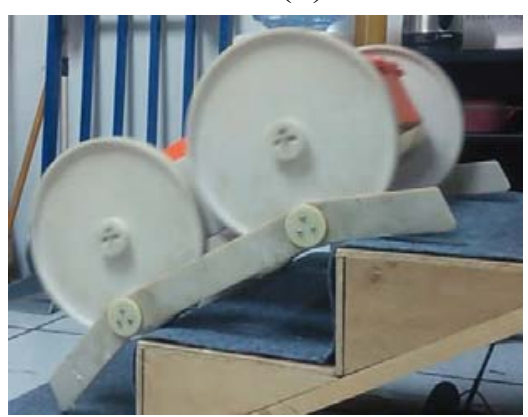

(d)

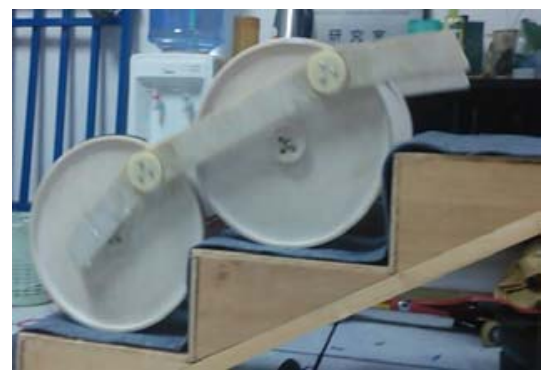

(f)

Fig. 7 stair climbing locomotion

According to the 50 times regular terrain walking; 100 times obstacle-overcoming and 50 times stair-climbing experiments, we test the times of singularity phenomenon. Experimental results' data is listed in the following Table 1. 
Table 1: Robots' testing results

\begin{tabular}{l|l|l|l|l|l|l|l|l|l}
\hline \multicolumn{3}{c|}{ Connecting-rod wheeled robot } & \multicolumn{2}{c}{ Multiphase connecting-rod wheeled robot } \\
\hline \multicolumn{3}{c}{ Walking terrain } & \multicolumn{2}{c}{$\begin{array}{c}\text { Motor } \\
\text { parameters }\end{array}$} & \multicolumn{3}{c|}{ Walking terrain } & \multicolumn{2}{c}{$\begin{array}{c}\text { Motor } \\
\text { parameters }\end{array}$} \\
\hline regular & obstacle & stair & $\begin{array}{l}\text { torque } \\
(\mathrm{Nm})\end{array}$ & $\begin{array}{l}\omega(\mathrm{rad} / \mathrm{m} \\
\text { in) }\end{array}$ & regular & obstacle & stair & $\begin{array}{l}\text { torque } \\
\text { (Nm) }\end{array}$ & $\begin{array}{l}\omega(\mathrm{rad} / \mathrm{m} \\
\text { in) }\end{array}$ \\
\hline 29 & 89 & 33 & 1.72 & 120 & 0 & 0 & 0 & 1.72 & 120 \\
\hline
\end{tabular}

\section{Summary}

This paper has analyzed the reason of the singularity phenomenon in the process of connecting-rod wheeled robot's movement and proposed a multiphase connecting-rod structure. By conducting experiments, we have proved that the robot which is designed with this structure can completely avoid the singularity phenomenon and its obstacle-overcoming ability is well. We currently perfect its structure and algorithm and expect it can be used in the rescue and detection field.

\section{Acknowledgement}

This work is financially supported by "Innovative Research Project for Postgraduate Students of Chongqing, China"(CYS2015050) and "Fundamental Research Funds for the Central Universities of China"(XDJK2016D022).

\section{Reference}

[1] Chen Diansheng, Huang Yu Wang, Tianmiao. Obstacle climbing analysis and simulation of wheel-legged robot[J]. Journal of Beijing University of Aeronautics and Astronautics,2009, 35 (3): $371 \sim 375$.

[2] Li Junke. wheel ski style robot design and analysis [D]. Chongqing: Southwestern University, 2013

[3] Wan Kegang. The static Analysis of Four-bar-mechanism in a plane[J]. Journal of Jiangxi Polytechnic University, 1986,8 (1): 58 62.

[4] Guan Lizhi. The Analysis of the Vector Equation of Four-bar-mechanism in plane[J]. Journal of Nanchang University(Engineering \& Technology),1997,19(2):29 31.

[5] Yue Dongpen. Auto machinery foundation[M]. Beijing: Higher Education Press,2013.

[6] $\mathrm{Hu}$ Xiang. Small robot climbing the ladder of continuous[D]. Chongqing: Southwestern University, 2015.

[7] Shen-Chiang Chen, Chih-Chung Ko, Cheng-Hsin Li, Pei-Chun Lin. Stair Climbing in a Quadruped Robot.[J]. International Journal of Automation and Smart Technology 2012,2(1):11-20

[8] ByungHoon Seo, HyunGyu Kim, MinHyeok Kim, Kyungmin Jeong. Flipbot:A New Field Robotic Platform for Fast Stair Climbing[J].International Journal of Precision Engineering and Manufacturing 2013,14(11):1909-1914. 